\title{
Some statistical approximation properties of Kantorovich-type $q$-Bernstein-Stancu operators
}

\author{
Mei-Ying Ren ${ }^{1 *}$ and Xiao-Ming Zeng ${ }^{2}$
}

${ }^{\text {"Correspondence: }}$

npmeiyingr@163.com

${ }^{1}$ Department of Mathematics and

Computer Science, Wuyi University,

Wuyishan, 354300, China

Full list of author information is

available at the end of the article

\section{Introduction}

In 1997, Phillips [1] introduced and studied $q$ analogue of Bernstein polynomials. During the last decade, the applications of $q$-calculus in the approximation theory have become one of the main area of research, $q$-calculus has been extensively used for constructing various generalizations of many classical approximation processes. It is well known that many $q$-extensions of the classical objects arising in the approximation theory have been recently introduced and studied (e.g., see [2-8]). Recently the statistical approximation properties have also been investigated for $q$-analogue polynomials. For instance, in [9] Kantorovich-type $q$-Bernstein operators; in [10] $q$-Baskakov-Kantorovich operators; in [11] Kantorovich-type $q$-Szász-Mirakjan operators; in [12] $q$-Bleimann, Butzer and Hahn operators; in [13] $q$-analogue of $\mathrm{MKZ}$ operators were introduced and their statistical approximation properties were studied.

The goal of this paper is to introduce two kinds of new Kantorovich-type $q$-BernsteinStancu operators and to study the statistical approximation properties of these operators with the help of the Korovkin-type approximation theorem. We also estimate the rate of statistical convergence of the mentioned sequences of operators to the appropriate function $f$, respectively.

Before proceeding further, let us give some basic definitions and notations from $q$-calculus. Details on $q$-integers can be found in $[14,15]$.

Let $q>0$, for each nonnegative integer $k$, the $q$-integer $[k]_{q}$ and the $q$-factorial $[k]_{q}$ ! are defined by

$$
[k]_{q}:= \begin{cases}\left(1-q^{k}\right) /(1-q), & q \neq 1, \\ k, & q=1\end{cases}
$$


and

$$
[k]_{q} !:= \begin{cases}{[k]_{q}[k-1]_{q} \cdots[1]_{q},} & k \geq 1, \\ 1, & k=0,\end{cases}
$$

respectively.

Then for $q>0$ and integers $n, k, n \geq k \geq 0$, we have

$$
[k+1]_{q}=1+q[k]_{q} \text { and }[k]_{q}+q^{k}[n-k]_{q}=[n]_{q} .
$$

For the integers $n, k, n \geq k \geq 0$, the $q$-binomial coefficient is defined by

$$
\left[\begin{array}{l}
n \\
k
\end{array}\right]_{q}:=\frac{[n]_{q} !}{[k]_{q} ![n-k]_{q} !}
$$

For an arbitrary function $f(x)$, the $q$-differential is given by

$$
d_{q} f(x)=f(q x)-f(x)
$$

The $q$-Jackson integral in the interval $[0, b]$ is defined as

$$
\int_{0}^{b} f(t) d_{q} t=(1-q) b \sum_{j=0}^{\infty} f\left(q^{j} b\right) q^{j}, \quad 0<q<1,
$$

provided that sums converge absolutely.

Suppose $0<a<b$. The $q$-Jackson integral in a generic interval $[a, b]$ is defined as

$$
\int_{a}^{b} f(t) d_{q} t=\int_{0}^{b} f(t) d_{q} t-\int_{0}^{a} f(t) d_{q} t, \quad 0<q<1 .
$$

\section{Construction of the operators}

In this part, we first construct the Kantorovich-type $q$-Bernstein-Stancu operators as follows.

Definition 1 Let $f$ be a $q$-integrable function on $[0,1]$, for $0 \leq \alpha \leq \beta, x \in[0,1], n \in \mathbf{N}$, $0<q<1$, we define the Kantorovich-type $q$-Bernstein-Stancu operators by

$$
S_{n, q}^{(\alpha, \beta)}(f ; x)=\left([n+1]_{q}+\beta\right) \sum_{k=0}^{n} q^{-k} p_{n, k}(q ; x) \int_{\frac{[k]_{q}+\alpha}{[n+1]_{q}+\beta}}^{\frac{[k+1]_{q}+\alpha}{[n+1)+\beta}} f(t) d_{q} t
$$

where

$$
p_{n, k}(q ; x)=\left[\begin{array}{l}
n \\
k
\end{array}\right]_{q} x^{k} \prod_{s=0}^{n-k-1}\left(1-q^{s} x\right)=\left[\begin{array}{l}
n \\
k
\end{array}\right]_{q} x^{k}(1-x)_{q}^{n-k} .
$$

In the following we give some lemmas. 
Lemma 1 For $S_{n, q}^{(\alpha, \beta)}\left(t^{i} ; x\right), i=0,1,2,0<q<1$, we have

$$
\begin{aligned}
S_{n, q}^{(\alpha, \beta)}(1 ; x)= & 1, \\
S_{n, q}^{(\alpha, \beta)}(t ; x)= & \frac{[n]_{q}}{[n+1]_{q}+\beta} x+\frac{1+2 \alpha}{[2]_{q}\left([n+1]_{q}+\beta\right)}, \\
S_{n, q}^{(\alpha, \beta)}\left(t^{2} ; x\right)= & \frac{q[n]_{q}[n-1]_{q}}{\left([n+1]_{q}+\beta\right)^{2}} x^{2}+\frac{\left(2+3 q+q^{2}+3 \alpha+3 \alpha q\right)[n]_{q}}{[3]_{q}\left([n+1]_{q}+\beta\right)^{2}} x \\
& +\frac{1+3 \alpha+3 \alpha^{2}}{[3]_{q}\left([n+1]_{q}+\beta\right)^{2}} .
\end{aligned}
$$

Proof For $i=0$, since $\sum_{k=0}^{n} p_{n, k}(q ; x)=1, \int_{\frac{[k] q+\alpha}{[n+1]_{q}+\beta}}^{\frac{[k+1]_{q}+\alpha}{[n+1]_{q}+\beta}} d_{q} t=\frac{q^{k}}{[n+1]_{q}+\beta}$, so Eq. (2) holds.

For $i=1$

$$
\begin{aligned}
S_{n, q}^{(\alpha, \beta)}(t ; x) & =\left([n+1]_{q}+\beta\right) \sum_{k=0}^{n} q^{-k} p_{n, k}(q ; x) \int_{\frac{[k]_{q}+\alpha}{[n+1]_{q}+\beta}}^{\frac{[k+1]_{q}+\alpha}{[n+1+\beta}} t d_{q} t \\
& =\sum_{k=0}^{n} p_{n, k}(q ; x) \frac{1+2 \alpha+[2]_{q}[k]_{q}}{[2]_{q}\left([n+1]_{q}+\beta\right)} \\
& =\frac{[n]_{q}}{[n+1]_{q}+\beta} x+\frac{1+2 \alpha}{[2]_{q}\left([n+1]_{q}+\beta\right)} .
\end{aligned}
$$

For $i=2$,

$$
\begin{aligned}
S_{n, q}^{(\alpha, \beta)}\left(t^{2} ; x\right)= & \left([n+1]_{q}+\beta\right) \sum_{k=0}^{n} q^{-k} p_{n, k}(q ; x) \int_{\frac{[k] q+\alpha}{[n+1]_{q}+\beta}}^{\frac{[k+1]_{q}+\alpha}{[n+1]_{q}+\beta}} t^{2} d_{q} t \\
= & \frac{[n]_{q}}{[3]_{q}\left([n+1]_{q}+\beta\right)^{2}} \\
& \times \sum_{k=0}^{n} p_{n, k}(q ; x) \frac{1+3 \alpha+3 \alpha^{2}+\left(1+2 q+3 \alpha[2]_{q}\right)[k]_{q}+[3]_{q}[k]_{q}^{2}}{[n]_{q}} \\
= & \frac{q[n]_{q}[n-1]_{q}}{\left([n+1]_{q}+\beta\right)^{2}} x^{2}+\frac{\left(2+3 q+q^{2}+3 \alpha+3 \alpha q\right)[n]_{q}}{[3]_{q}\left([n+1]_{q}+\beta\right)^{2}} x \\
& +\frac{1+3 \alpha+3 \alpha^{2}}{[3]_{q}\left([n+1]_{q}+\beta\right)^{2}} .
\end{aligned}
$$

Lemma 2 For $n \in \mathbf{N}, x \in[0,1], 0<q<1,0 \leq \alpha \leq \beta$, we have

$$
S_{n, q}^{(\alpha, \beta)}\left((t-x)^{2} ; x\right) \leq \frac{13(1+\beta)^{2}}{[n+1]_{q}+\beta}\left(\frac{1}{4}+\frac{1}{[n+1]_{q}+\beta}\right) .
$$

Proof In view of Lemma 1 and $\max _{x \in[0,1]} x(1-x)=\frac{1}{4}$, by a simple computation, we have

$$
\begin{aligned}
& S_{n, q}^{(\alpha, \beta)}\left((t-x)^{2} ; x\right) \\
& \quad=S_{n, q}^{(\alpha, \beta)}\left(t^{2} ; x\right)-2 x S_{n, q}^{(\alpha, \beta)}(t ; x)+x^{2} S_{n, q}^{(\alpha, \beta)}(1 ; x)
\end{aligned}
$$




$$
\begin{aligned}
= & \frac{1+3 \alpha+3 \alpha^{2}}{[3]_{q}\left([n+1]_{q}+\beta\right)^{2}}+\frac{x}{[2]_{q}[3]_{q}\left([n+1]_{q}+\beta\right)^{2}} \\
& \times\left[[2]_{q}\left(2+3 q+q^{2}+3 \alpha+3 \alpha q\right)[n]_{q}-2(1+2 \alpha)[3]_{q}\left([n+1]_{q}+\beta\right)\right] \\
& +\frac{q[n]_{q}[n-1]_{q}-2[n]_{q}\left([n+1]_{q}+\beta\right)+\left([n+1]_{q}+\beta\right)^{2}}{\left([n+1]_{q}+\beta\right)^{2}} x^{2} \\
\leq & \frac{13(1+\beta)^{2}}{[n+1]_{q}+\beta}\left(x(1-x)+\frac{1}{[n+1]_{q}+\beta}\right) \\
\leq & \frac{13(1+\beta)^{2}}{[n+1]_{q}+\beta}\left(\frac{1}{4}+\frac{1}{[n+1]_{q}+\beta}\right)
\end{aligned}
$$

Lemma 3 ([9]) Let $0 \leq a<b, 0<q<1$, and let $f$ be a positive function defined on the interval $[0, b]$. Iff is monotone increasing on $[0, b]$, then $\int_{a}^{b} f(t) d_{q} t \geq 0$ in this interval.

It is clear that the operator $S_{n, q}^{(\alpha, \beta)}(f ; x)$ is a linear and positive operator for any monotone increasing function $f \in[0,1]$.

Remark 1 To guarantee the positivity of $S_{n, q}^{(\alpha, \beta)}(f ; x), f$ must be a monotone increasing function on the interval $[0,1]$. But for the function $f$ this condition is strong. In order to solve the problems, a special type of $q$-integral, which is the Riemann-type $q$-integral, is defined by Marinković et al. [16].

Definition 2 ([16]) Let $0<a<b, 0<q<1$. The Riemann-type $q$-integral is defined as

$$
R_{q}(f ; a, b)=\int_{a}^{b} f(t) d_{q}^{R} t=(1-q)(b-a) \sum_{j=0}^{\infty} f\left(a+(b-a) q^{j}\right) q^{j}
$$

provided the sums converge absolutely.

We now redefine $S_{n, q}^{(\alpha, \beta)}(f ; x)$ by putting the Riemann-type $q$-integral into the operators instead of the general $q$-integral as

$$
\widetilde{S}_{n, q}^{(\alpha, \beta)}(f ; x)=\left([n+1]_{q}+\beta\right) \sum_{k=0}^{n} q^{-k} p_{n, k}(q ; x) \int_{\frac{[k] q+\alpha}{[n+1]_{q}+\beta}}^{\frac{[k+1] q+\alpha}{[n+1] q+\beta}} f(t) d_{q}^{R} t
$$

where $f$ is a Riemann-type $q$-integrable function on $[0,1]$.

Let us give some lemmas as follows.

Lemma 4 Let $0<q<1,0 \leq \alpha \leq \beta$, then $\left\{\widetilde{S}_{n, q}^{(\alpha, \beta)}(f ; x)\right\}$ is a linear and positive operator.

Proof The proof is clear, so we omit it.

Lemma 5 For $\widetilde{S}_{n, q}^{(\alpha, \beta)}\left(t^{i} ; x\right), i=0,1,2,0<q<1,0 \leq \alpha \leq \beta$, we have

$$
\begin{aligned}
& \widetilde{S}_{n, q}^{(\alpha, \beta)}(1 ; x)=1, \\
& \widetilde{S}_{n, q}^{(\alpha, \beta)}(t ; x)=\frac{2 q[n]_{q}}{[2]_{q}\left([n+1]_{q}+\beta\right)} x+\frac{1+[2]_{q} \alpha}{[2]_{q}\left([n+1]_{q}+\beta\right)},
\end{aligned}
$$




$$
\begin{aligned}
\widetilde{S}_{n, q}^{(\alpha, \beta)}\left(t^{2} ; x\right)= & \frac{q[n]_{q}[n-1]_{q}}{\left([n+1]_{q}+\beta\right)^{2}}\left(1+\frac{2(q-1)}{[2]_{q}}+\frac{(q-1)^{2}}{[3]_{q}}\right) x^{2} \\
& +\frac{[n]_{q}}{\left([n+1]_{q}+\beta\right)^{2}}\left(1+2 \alpha+\frac{2(q-1)(1+\alpha)}{[2]_{q}}+\frac{2}{[2]_{q}}\right. \\
& \left.+\frac{2(q-1)}{[3]_{q}}+\frac{(q-1)^{2}}{[3]_{q}}\right) x+\frac{1}{\left([n+1]_{q}+\beta\right)^{2}}\left(\frac{1}{[3]_{q}}+\frac{2 \alpha}{[2]_{q}}+\alpha^{2}\right) .
\end{aligned}
$$

Proof By Definition 2, we have

$$
\begin{aligned}
& \int_{\frac{[k] q+\alpha}{\left[n+1 q_{q}+\beta\right.}}^{\frac{[k+1] q+\alpha}{[n+1]+\beta}} d_{q}^{R} t=\frac{q^{k}}{[n+1]_{q}+\beta}, \\
& \int_{\frac{[k] q+\alpha}{\left[n+1 q_{q}+\beta\right.}}^{\frac{[k+1]_{q}+\alpha}{[n+1]_{q}+\beta}} t d_{q}^{R} t=\frac{1}{\left([n+1]_{q}+\beta\right)^{2}}\left\{q^{k}\left([k]_{q}+\alpha\right)+\frac{q^{2 k}}{[2]_{q}}\right\}, \\
& \int_{\frac{\left[k q_{q}+\alpha\right.}{[n+1]_{q}+\beta}}^{\frac{[k+1]_{q}+\alpha}{[n+1}} t^{2} d_{q}^{R} t=\frac{1}{\left([n+1]_{q}+\beta\right)^{3}}\left\{q^{k}\left([k]_{q}+\alpha\right)^{2}+\frac{2 q^{2 k}}{[2]_{q}}\left([k]_{q}+\alpha\right)+\frac{q^{3 k}}{[3]_{q}}\right\} \text {. }
\end{aligned}
$$

Hence, by using the equality $\sum_{k=0}^{n} p_{n, k}(q ; x)=1$ and Eq. (10), we get

$$
\widetilde{S}_{n, q}^{(\alpha, \beta)}(1 ; x)=1
$$

By using Eq. (11) and the equality $q^{k}=(q-1)[k]_{q}+1$, we have

$$
\begin{aligned}
\widetilde{S}_{n, q}^{(\alpha, \beta)}(t ; x) & =\frac{1}{[n+1]_{q}+\beta} \sum_{k=0}^{n} p_{n, k}(q ; x)\left\{[k]_{q}+\alpha+\frac{(q-1)[k]_{q}+1}{[2]_{q}}\right\} \\
& =\frac{2 q[n]_{q}}{[2]_{q}\left([n+1]_{q}+\beta\right)} x+\frac{1+[2]_{q} \alpha}{[2]_{q}\left([n+1]_{q}+\beta\right)} .
\end{aligned}
$$

By using Eq. (12) and the equality $q^{k}=(q-1)[k]_{q}+1$, we have

$$
\begin{aligned}
\widetilde{S}_{n, q}^{(\alpha, \beta)}\left(t^{2} ; x\right) & \frac{1}{\left([n+1]_{q}+\beta\right)^{2}} \sum_{k=0}^{n} p_{n, k}(q ; x)\left\{\left([k]_{q}+\alpha\right)^{2}+\frac{2 q^{k}}{[2]_{q}}\left([k]_{q}+\alpha\right)+\frac{q^{2 k}}{[3]_{q}}\right\} \\
= & \frac{[n]_{q}}{\left([n+1]_{q}+\beta\right)^{2}} \sum_{k=0}^{n} \frac{p_{n, k}(q ; x)}{[n]_{q}}\left\{\frac{1}{[3]_{q}}+\frac{2 \alpha}{[2]_{q}}+\alpha^{2}+\left(2 \alpha+\frac{2(q-1) \alpha}{[2]_{q}}\right.\right. \\
& \left.\left.+\frac{2}{[2]_{q}}+\frac{2(q-1)}{[3]_{q}}\right)[k]_{q}+\left(1+\frac{2(q-1)}{[2]_{q}}+\frac{(q-1)^{2}}{[3]_{q}}\right)[k]_{q}^{2}\right\} \\
= & \frac{q[n]_{q}[n-1]_{q}}{\left([n+1]_{q}+\beta\right)^{2}}\left(1+\frac{2(q-1)}{[2]_{q}}+\frac{(q-1)^{2}}{[3]_{q}}\right) x^{2} \\
& +\frac{[n]_{q}}{\left([n+1]_{q}+\beta\right)^{2}}\left(1+2 \alpha+\frac{2(q-1)(1+\alpha)}{[2]_{q}}+\frac{2}{[2]_{q}}+\frac{2(q-1)}{[3]_{q}}\right. \\
& \left.+\frac{(q-1)^{2}}{[3]_{q}}\right) x+\frac{1}{\left([n+1]_{q}+\beta\right)^{2}}\left(\frac{1}{[3]_{q}}+\frac{2 \alpha}{[2]_{q}}+\alpha^{2}\right) .
\end{aligned}
$$


Lemma 6 For $n \in \mathbf{N}, x \in[0,1], 0<q<1,0 \leq \alpha \leq \beta$, we have

$$
\widetilde{S}_{n, q}^{(\alpha, \beta)}\left((t-x)^{2} ; x\right) \leq 2(1-q)^{2}+\frac{1}{[n+1]_{q}+\beta}\left(3+\frac{(1+\alpha)^{2}+(1+\beta)^{2}}{[n+1]_{q}+\beta}\right) .
$$

Proof In view of Lemma 5, by a simple computation, we have

$$
\begin{aligned}
& \widetilde{S}_{n, q}^{(\alpha, \beta)}\left((t-x)^{2} ; x\right) \\
&=\widetilde{S}_{n, q}^{(\alpha, \beta)}\left(t^{2} ; x\right)-2 x \widetilde{S}_{n, q}^{(\alpha, \beta)}(t ; x)+x^{2} \widetilde{S}_{n, q}^{(\alpha, \beta)}(1 ; x) \\
&=\left[\frac{q[n]_{q}[n-1]_{q}}{\left([n+1]_{q}+\beta\right)^{2}}\left(1+\frac{2(q-1)}{[2]_{q}}+\frac{(q-1)^{2}}{[3]_{q}}\right)-\frac{4 q[n]_{q}}{[2]_{q}\left([n+1]_{q}+\beta\right)}+1\right] x^{2} \\
&+\frac{[n]_{q}}{\left([n+1]_{q}+\beta\right)^{2}}\left(1+2 \alpha+\frac{2(q-1)(1+\alpha)}{[2]_{q}}+\frac{2}{[2]_{q}}+\frac{2(q-1)}{[3]_{q}}\right. \\
&\left.+\frac{(q-1)^{2}}{[3]_{q}}\right) x-\frac{2\left(1+[2]_{q} \alpha\right)}{[2]_{q}\left([n+1]_{q}+\beta\right)} x \\
&+\frac{1}{\left([n+1]_{q}+\beta\right)^{2}}\left(\frac{1}{[3]_{q}}+\frac{2 \alpha}{[2]_{q}}+\alpha^{2}\right) \\
& \leq\left(2(1-q)^{2}+\frac{(1+\beta)^{2}}{\left([n+1]_{q}+\beta\right)^{2}}\right) x^{2}+\frac{3}{[n+1]_{q}+\beta} x+\frac{(1+\alpha)^{2}}{\left([n+1]_{q}+\beta\right)^{2}} \\
& \leq 2(1-q)^{2}+\frac{1}{[n+1]_{q}+\beta}\left(3+\frac{(1+\alpha)^{2}+(1+\beta)^{2}}{[n+1]_{q}+\beta}\right) .
\end{aligned}
$$

\section{Statistical approximation of Korovkin type}

Now, let us recall the concept of statistical convergence which was introduced by Fast [17].

Let set $K \subseteq \mathbf{N}$ and $K_{n}=\{k \leq n: k \in K\}$, the natural density of $K$ is defined by $\delta(K):=$ $\lim _{n \rightarrow \infty} \frac{1}{n}\left|K_{n}\right|$ if the limit exists (see [18]), where $\left|K_{n}\right|$ denotes the cardinality of the set $K_{n}$.

A sequence $x=\left\{x_{k}\right\}$ is called statistically convergent to a number $L$ if for every $\varepsilon>0$, $\delta\left\{k \in \mathbf{N}:\left|x_{k}-L\right| \geq \varepsilon\right\}=0$. This convergence is denoted as $s t-\lim _{k} x_{k}=L$.

Note that any convergent sequence is statistically convergent, but not conversely. Details can be found in [19].

In approximation theory, the concept of statistical convergence was used by Gadjiev and Orhan [20]. They proved the following Bohman-Korovkin type approximation theorem for statistical convergence.

Theorem 1 ([20]) If the sequence of linear positive operators $A_{n}: C[a, b] \rightarrow C[a, b]$ satisfies the conditions

$$
s t-\lim _{n}\left\|A_{n}\left(e_{v} ; \cdot\right)-e_{v}\right\|_{C[a, b]}=0
$$

for $e_{v}(t)=t^{v}, v=0,1,2$. Then, for any $f \in C[a, b]$,

$$
s t-\lim _{n}\left\|A_{n}(f ; \cdot)-f\right\|_{C[a, b]}=0 .
$$

Theorem 2 Let $q=\left\{q_{n}\right\}, 0<q_{n}<1$, be a sequence satisfying the following condition

$$
s t-\lim _{n} q_{n}=1, \quad s t-\lim _{n} q_{n}^{n}=a \quad(a<1) \quad \text { and } \quad s t-\lim _{n} \frac{1}{[n]_{q_{n}}}=0,
$$


then for any monotone increasing function $f \in C[0,1]$, we have

$$
s t-\lim _{n}\left\|S_{n, q_{n}}^{(\alpha, \beta)}(f ; \cdot)-f\right\|_{C[0,1]}=0 .
$$

Proof From Theorem 1, it is enough to prove that $s t-\lim _{n}\left\|S_{n, q_{n}}^{(\alpha, \beta)}\left(e_{v} ; \cdot\right)-e_{v}\right\|_{C[0,1]}=0$ for $e_{v}(t)=t^{v}, v=0,1,2$.

From Eq. (2), we can easily get

$$
s t-\lim _{n}\left\|S_{n, q_{n}}^{(\alpha, \beta)}\left(e_{0} ; \cdot\right)-e_{0}\right\|_{C[0,1]}=0 \text {. }
$$

From Eq. (3), we have

$$
S_{n, q_{n}}^{(\alpha, \beta)}\left(e_{1} ; x\right)-e_{1}(x)=\left(\frac{[n]_{q_{n}}}{[n+1]_{q_{n}}+\beta}-1\right) x+\frac{1+2 \alpha}{[2]_{q_{n}}\left([n+1]_{q_{n}}+\beta\right)} .
$$

In view of $[n+1]_{q_{n}}=1+q_{n}[n]_{q_{n}}$, for $\beta>0$ we have

$$
\left\|S_{n, q_{n}}^{(\alpha, \beta)}\left(e_{1} ; \cdot\right)-e_{1}\right\|_{C[0,1]} \leq\left(1-q_{n}\right)+\left(1+\beta+\frac{1+2 \alpha}{[2]_{q_{n}}}\right) \frac{1}{[n]_{q_{n}}} .
$$

Now, for every given $\varepsilon>0$, let us define the following sets:

$$
\begin{aligned}
& U=\left\{k:\left\|S_{n, q_{k}}^{(\alpha, \beta)}\left(e_{1} ; \cdot\right)-e_{1}\right\|_{C[0,1]} \geq \varepsilon\right\}, \\
& U_{1}=\left\{k: 1-q_{k} \geq \frac{\varepsilon}{2}\right\}, \\
& U_{2}=\left\{k:\left(1+\beta+\frac{1+2 \alpha}{[2]_{q_{k}}}\right) \frac{1}{[k]_{q_{k}}} \geq \frac{\varepsilon}{2}\right\} .
\end{aligned}
$$

From inequality (15), one can see that $U \subseteq U_{1} \cup U_{2}$, so we have

$$
\begin{aligned}
\delta\left\{k \leq n:\left\|S_{n, q_{k}}^{(\alpha, \beta)}\left(e_{1} ; \cdot\right)-e_{1}\right\|_{C[0,1]} \geq \varepsilon\right\} \\
\leq \delta\left\{k \leq n: 1-q_{k} \geq \frac{\varepsilon}{2}\right\} \\
\quad+\delta\left\{k \leq n:\left(1+\beta+\frac{1+2 \alpha}{[2]_{q_{k}}}\right) \frac{1}{[k]_{q_{k}}} \geq \frac{\varepsilon}{2}\right\} .
\end{aligned}
$$

By condition (13), it is clear that $s t-\lim _{n}\left(1-q_{n}\right)=0$, st $-\lim _{n}\left(1+\beta+\frac{1+2 \alpha}{[2]_{q_{n}}}\right) \frac{1}{[n]_{q_{n}}}=0$. So we have

$$
s t-\lim _{n}\left\|S_{n, q_{n}}^{(\alpha, \beta)}\left(e_{1} ; \cdot\right)-e_{1}\right\|_{C[0,1]}=0 .
$$

In view of Eq. (4) and the equality $[n+1]_{q_{n}}=1+q_{n}[n]_{q_{n}}$, by a simple computation, for $\beta>0$ we have

$$
\left\|S_{n, q_{n}}^{(\alpha, \beta)}\left(e_{2} ; \cdot\right)-e_{2}\right\|_{C[0,1]} \leq \frac{11+9 \alpha+3 \alpha^{2}+4 \beta+\beta^{2}}{[n]_{q_{n}}} .
$$


Now, for every given $\varepsilon>0$, let us define the following sets:

$$
\begin{aligned}
& T=\left\{k:\left\|S_{n, q_{k}}^{(\alpha, \beta)}\left(e_{2} ; \cdot\right)-e_{2}\right\|_{C[0,1]} \geq \varepsilon\right\}, \\
& T_{1}=\left\{k: \frac{11+9 \alpha+3 \alpha^{2}+4 \beta+\beta^{2}}{[k]_{q_{k}}} \geq \varepsilon\right\} .
\end{aligned}
$$

It is clear that $T \subseteq T_{1}$, so we get

$$
\delta\left\{k \leq n:\left\|S_{n, q_{k}}^{(\alpha, \beta)}\left(e_{2} ; \cdot\right)-e_{2}\right\|_{C[0,1]} \geq \varepsilon\right\} \leq \delta\left\{k \leq n: \frac{11+9 \alpha+3 \alpha^{2}+4 \beta+\beta^{2}}{[k]_{q_{k}}} \geq \varepsilon\right\} .
$$

By condition (13), we have

$$
\text { st }-\lim _{n} \frac{11+9 \alpha+3 \alpha^{2}+4 \beta+\beta^{2}}{[n]_{q_{n}}}=0
$$

so, we can get

$$
s t-\lim _{n}\left\|S_{n, q_{n}}^{(\alpha, \beta)}\left(e_{2} ; \cdot\right)-e_{2}\right\|_{C[0,1]}=0 .
$$

In view of Eqs. (14), (16) and (17), the proof is complete.

Theorem 3 Let $q=\left\{q_{n}\right\}, 0<q_{n}<1$, be a sequence satisfying condition (13), then for all $f \in C[0,1]$, we have st $-\lim _{n}\left\|\widetilde{S}_{n, q_{n}}^{(\alpha, \beta)}(f ; \cdot)-f\right\|_{C[0,1]}=0$.

Proof From Theorem 1, it is enough to prove that $s t-\lim _{n}\left\|\widetilde{S}_{n, q_{n}}^{(\alpha, \beta)}\left(e_{v} ; \cdot\right)-e_{v}\right\|_{C[0,1]}=0$, for $e_{v}(t)=t^{v}, v=0,1,2$.

From Eq. (7), we can easily get

$$
s t-\lim _{n}\left\|\widetilde{S}_{n, q_{n}}^{(\alpha, \beta)}\left(e_{0} ; \cdot\right)-e_{0}\right\|_{C[0,1]}=0 .
$$

From Eq. (8), we have

$$
\widetilde{S}_{n, q_{n}}^{(\alpha, \beta)}\left(e_{1} ; x\right)-e_{1}(x)=\left(\frac{2 q_{n}[n]_{q_{n}}}{[2]_{q_{n}}\left([n+1]_{q_{n}}+\beta\right)}-1\right) x+\frac{1+[2]_{q_{n}} \alpha}{[2]_{q_{n}}\left([n+1]_{q_{n}}+\beta\right)} .
$$

In view of $[n+1]_{q_{n}}=1+q_{n}[n]_{q_{n}}$, for $\beta>0$ we have

$$
\left\|\widetilde{S}_{n, q_{n}}^{(\alpha, \beta)}\left(e_{1} ; \cdot\right)-e_{1}\right\|_{C[0,1]} \leq\left(1-q_{n}\right)+\left[\left(1+q_{n}\right)(1+\beta)+1+[2]_{q_{n}} \alpha\right] \frac{1}{[n]_{q_{n}}} .
$$

Now, for every given $\varepsilon>0$, let us define the following sets:

$$
\begin{aligned}
& \widetilde{U}=\left\{k:\left\|\widetilde{S}_{n, q_{k}}^{(\alpha, \beta)}\left(e_{1} ; \cdot\right)-e_{1}\right\|_{C[0,1]} \geq \varepsilon\right\}, \\
& \widetilde{U}_{1}=\left\{k: 1-q_{k} \geq \frac{\varepsilon}{2}\right\}, \\
& \widetilde{U}_{2}=\left\{k:\left[\left(1+q_{k}\right)(1+\beta)+1+[2]_{q_{k}} \alpha\right] \frac{1}{[k]_{q_{k}}} \geq \frac{\varepsilon}{2}\right\} .
\end{aligned}
$$


From inequality (19), one can see that $\widetilde{U} \subseteq \widetilde{U}_{1} \cup \widetilde{U}_{2}$, so we have

$$
\begin{aligned}
\delta\left\{k \leq n:\left\|\widetilde{S}_{n, q_{k}}^{(\alpha, \beta)}\left(e_{1} ; \cdot\right)-e_{1}\right\|_{C[0,1]} \geq \varepsilon\right\} \\
\quad \leq \delta\left\{k \leq n: 1-q_{k} \geq \frac{\varepsilon}{2}\right\} \\
\quad+\delta\left\{k \leq n:\left[\left(1+q_{k}\right)(1+\beta)+1+[2]_{q_{k}} \alpha\right] \frac{1}{[k]_{q_{k}}} \geq \frac{\varepsilon}{2}\right\} .
\end{aligned}
$$

By condition (13), it is clear that

$$
s t-\lim _{n}\left(1-q_{n}\right)=0, \quad s t-\lim _{n}\left[\left(1+q_{n}\right)(1+\beta)+1+[2]_{q_{n}} \alpha\right] \frac{1}{[n]_{q_{n}}}=0 .
$$

So we have

$$
s t-\lim _{n}\left\|\widetilde{S}_{n, q_{n}}^{(\alpha, \beta)}\left(e_{1} ; \cdot\right)-e_{1}\right\|_{C[0,1]}=0 .
$$

In view of Eq. (9) and the equality $[n+1]_{q_{n}}=1+q_{n}[n]_{q_{n}}$, for $\beta>0$ and $0<q_{n}<1$, by a simple computation, we have

$$
\left\|\widetilde{S}_{n, q_{n}}^{(\alpha, \beta)}\left(e_{2} ; \cdot\right)-e_{2}\right\|_{C[0,1]} \leq 6\left(1-q_{n}\right)+\frac{11+6 \alpha+\alpha^{2}+6(1+\beta)(3+\beta)}{[n]_{q_{n}}} .
$$

Now, for every given $\varepsilon>0$, let us define the following sets:

$$
\begin{aligned}
& \widetilde{T}=\left\{k:\left\|\widetilde{S}_{n, q_{k}}^{(\alpha, \beta)}\left(e_{2} ; \cdot\right)-e_{2}\right\|_{C[0,1]} \geq \varepsilon\right\}, \\
& \widetilde{T}_{1}=\left\{k: 6\left(1-q_{k}\right) \geq \frac{\varepsilon}{2}\right\}, \\
& \widetilde{T}_{2}=\left\{k: \frac{11+6 \alpha+\alpha^{2}+6(1+\beta)(3+\beta)}{[k]_{q_{k}}} \geq \frac{\varepsilon}{2}\right\} .
\end{aligned}
$$

It is clear that $\widetilde{T} \subseteq \widetilde{T}_{1} \cup \widetilde{T}_{2}$, so we get

$$
\begin{aligned}
& \delta\left\{k \leq n:\left\|\widetilde{S}_{n, q_{k}}^{(\alpha, \beta)}\left(e_{2} ; \cdot\right)-e_{2}\right\|_{C[0,1]} \geq \varepsilon\right\} \\
& \leq \delta\left\{k \leq n: 6\left(1-q_{k}\right) \geq \frac{\varepsilon}{2}\right\} \\
&+\delta\left\{k \leq n: \frac{11+6 \alpha+\alpha^{2}+6(1+\beta)(3+\beta)}{[k]_{q_{k}}} \geq \frac{\varepsilon}{2}\right\} .
\end{aligned}
$$

By condition (13), we have

$$
s t-\lim _{n} 6\left(1-q_{n}\right)=0, \quad s t-\lim _{n} \frac{11+6 \alpha+\alpha^{2}+6(1+\beta)(3+\beta)}{[n]_{q_{n}}}=0 .
$$

So, we can get

$$
s t-\lim _{n}\left\|\widetilde{S}_{n, q_{n}}^{(\alpha, \beta)}\left(e_{2} ; \cdot\right)-e_{2}\right\|_{C[0,1]}=0 .
$$

In view of Eqs. (18), (20) and (21), the proof is complete. 


\section{Rates of statistical convergence}

Let $f \in C[0,1]$, for any $\delta>0$, the usual modulus of continuity for $f$ is defined as $\omega(f ; \delta)=$ $\sup _{0<h \leq \delta} \sup _{x, x+h \in[0,1]}|f(x+h)-f(x)|$.

For $f \in C[0,1]$ and any $t, x \in[0,1]$, we have $|f(t)-f(x)| \leq \omega(f,|t-x|)$, so for any $\delta>0$, we get

$$
\omega(f,|t-x|) \leq \begin{cases}\omega(f, \delta), & |t-x|<\delta \\ \omega\left(f, \frac{(t-x)^{2}}{\delta}\right), & |t-x| \geq \delta .\end{cases}
$$

In the light of $\omega(f ; \lambda \delta) \leq(1+\lambda) \omega(f ; \delta)$ for $\lambda>0$, it is clear that we have

$$
|f(t)-f(x)| \leq\left(1+\delta^{-2}(t-x)^{2}\right) \omega(f, \delta)
$$

for any $t, x \in[0,1]$ and $\delta>0$.

Next we will give the rates of convergence of both $S_{n, q_{n}}^{(\alpha, \beta)}(f ; x)$ and $\widetilde{S}_{n, q_{n}}^{(\alpha, \beta)}(f ; x)$ by means of modulus of continuity.

Theorem 4 Let $q=\left\{q_{n}\right\}, 0<q_{n}<1$ be a sequence satisfying condition (13), then for any monotone increasing function $f \in C[0,1]$, we have

$$
\left\|S_{n, q_{n}}^{(\alpha, \beta)}(f ; \cdot)-f\right\|_{C[0,1]} \leq 2 \omega\left(f ; \delta_{n}\right)
$$

where

$$
\delta_{n}=\left\{\frac{13(1+\beta)^{2}}{[n+1]_{q_{n}}+\beta}\left(\frac{1}{4}+\frac{1}{[n+1]_{q_{n}}+\beta}\right)\right\}^{1 / 2} .
$$

Proof Using the linearity and positivity of the operator $S_{n, q}^{(\alpha, \beta)}(f ; x)$, by Lemma 2 and inequality (22), we get

$$
\begin{aligned}
\left|S_{n, q}^{(\alpha, \beta)}(f ; x)-f(x)\right| \\
\quad \leq S_{n, q}^{(\alpha, \beta)}(|f(t)-f(x)| ; x) \\
\quad \leq\left(1+\delta^{-2} S_{n, q}^{(\alpha, \beta)}\left((t-x)^{2} ; x\right)\right) \omega(f, \delta) \\
\quad \leq\left[1+\delta^{-2} \frac{13(1+\beta)^{2}}{[n+1]_{q}+\beta}\left(\frac{1}{4}+\frac{1}{[n+1]_{q}+\beta}\right)\right] \omega(f, \delta) .
\end{aligned}
$$

Take $q=\left\{q_{n}\right\}, 0<q_{n}<1$, to be a sequence satisfying condition (13) and choose $\delta=\delta_{n}$ in (23), we have $\left|S_{n, q_{n}}^{(\alpha, \beta)}(f ; x)-f(x)\right| \leq 2 \omega\left(f ; \delta_{n}\right)$, which implies the proof is complete.

Theorem 5 Let $q=\left\{q_{n}\right\}, 0<q_{n}<1$, be a sequence satisfying condition (13), then for any $f \in C[0,1]$, we have $\left\|\widetilde{S}_{n, q_{n}}^{(\alpha, \beta)}(f ; \cdot)-f\right\|_{C[0,1]} \leq 2 \omega\left(f ; \delta_{n}\right)$, where

$$
\delta_{n}=\left\{2(1-q)^{2}+\frac{1}{[n+1]_{q_{n}}+\beta}\left(3+\frac{(1+\alpha)^{2}+(1+\beta)^{2}}{[n+1]_{q_{n}}+\beta}\right)\right\}^{1 / 2} .
$$


Proof Using the linearity and positivity of the operator $\widetilde{S}_{n, q}^{(\alpha, \beta)}(f ; x)$, by Lemma 6 and inequality (22), we get

$$
\begin{aligned}
\left|\widetilde{S}_{n, q}^{(\alpha, \beta)}(f ; x)-f(x)\right| \\
\quad \leq \widetilde{S}_{n, q}^{(\alpha, \beta)}(|f(t)-f(x)| ; x) \\
\quad \leq\left(1+\delta^{-2} \widetilde{S}_{n, q}^{(\alpha, \beta)}\left((t-x)^{2} ; x\right)\right) \omega(f, \delta) \\
\quad \leq\left\{1+\delta^{-2}\left[2(1-q)^{2}+\frac{1}{[n+1]_{q}+\beta}\left(3+\frac{(1+\alpha)^{2}+(1+\beta)^{2}}{[n+1]_{q}+\beta}\right)\right]\right\} \omega(f, \delta) .
\end{aligned}
$$

Take $q=\left\{q_{n}\right\}, 0<q_{n}<1$, to be a sequence satisfying condition (13) and choose $\delta=\delta_{n}$ in (24), we have $\left|\widetilde{S}_{n, q_{n}}^{(\alpha, \beta)}(f ; x)-f(x)\right| \leq 2 \omega\left(f ; \delta_{n}\right)$, which implies the proof is complete.

\section{Competing interests}

The authors declare that they have no competing interests.

\section{Authors' contributions}

All authors contributed equally and significantly in writing this article. All authors read and approved the final manuscript.

\section{Author details}

${ }^{1}$ Department of Mathematics and Computer Science, Wuyi University, Wuyishan, 354300, China. ${ }^{2}$ Department of Mathematics, Xiamen University, Xiamen, 361005, China.

\section{Acknowledgements}

The authors are most grateful to the editor and anonymous referee for careful reading of the manuscript and valuable suggestions which helped in improving an earlier version of this paper. This work is supported by the National Natural Science Foundation of China (Grant No. 61170324), the Class A Science and Technology Project of Education Department of Fujian Province of China (Grant No. JA12324), and the Natural Science Foundation of Fujian Province of China (Grant No. 2013J01017)

\section{Received: 9 August 2013 Accepted: 9 December 2013 Published: 06 Jan 2014}

\section{References}

1. Phillips, GM: Bernstein polynomials based on the q-integers. Ann. Numer. Math. 4, 511-518 (1997)

2. Aral, A, Doğru, O: Bleimann, Butzer, and Hahn operators based on the q-integers. J. Inequal. Appl. 2007, 079410 (2007). doi:10.1155/2007/79410

3. Gupta, V, Wang, HP: The rate of convergence of $q$-Durrmeyer operators for $0<q<1$. Math. Methods Appl. Sci. 31(16), 1946-1955 (2008)

4. Doğru, O, Gupta, V: Korovkin-type approximation properties of bivariate q-Meyer-König and Zeller operators. Calcolo 43(1), 51-63 (2012)

5. Aral, A, Gupta, V, Agarwal, RP: Applications of q-Calculus in Operator Theory. Springer, New York (2013)

6. Verma, DK, Agrawal, PN: Approximation by Baskakov-Durrmeyer-Stancu operators based on q-integers. Lobachevskii J. Math. 43(2), 187-196 (2013)

7. Ren, MY, Zeng, XM: Approximation by a kind of complex modified q-Durrmeyer type operators in compact disks. J. Inequal. Appl. 2012, 212 (2012). doi:10.1186/1029-242X-2012-212

8. Ren, MY, Zeng, XM: Approximation of the summation-integral-type q-Szász-Mirakjan operators. Abstr. Appl. Anal. 2012, 614810 (2012). doi:10.1155/2012/614810

9. Dalmanoǧlu, Ö, Doğru, O: On statistical approximation properties of Kantorovich type $q$-Bernstein operators. Math. Comput. Model. 52(5-6), 760-771 (2010)

10. Gupta, V, Radu, C: Statistical approximation properties of $q$-Baskokov-Kantorovich operators. Cent. Eur. J. Math. 7(4), 809-818 (2009)

11. Örkcü, M, Doğru, O: Weighted statistical approximation by Kantorovich type $q$-Szász-Mirakjan operators. Appl. Math Comput. 217(20), 7913-7919 (2011)

12. Ersan, S, Doğru, O: Statistical approximation properties of q-Bleimann, Butzer and Hahn operators. Math. Comput. Model. 49(7-8), 1595-1606 (2009)

13. Aktuglu, H, Ozarslan, MA, Duman, O: Matrix summability methods on the approximation of multivariate $q-M K Z$ operators. Bull. Malays. Math. Sci. Soc. 34(3), 465-474 (2011)

14. Kac, VG, Cheung, P: Quantum Calculus. Universitext. Springer, New York (2002)

15. Gasper, G, Rahman, M: Basic Hypergeometric Series. Encyclopedia of Mathematics and Its Applications, vol. 35. Cambridge University Press, Cambridge (1990)

16. Marinković, S, Rajković, P, Stanković, M: The inequalities for some types of $q$-integrals. Comput. Math. Appl. 56(10), 2490-2498 (2008)

17. Fast, H: Sur la convergence statistique. Colloq. Math. 2(3-4), 241-244 (1951) 
18. Niven, I, Zuckerman, HS, Montgomery, H: An Introduction to the Theory Numbers, 5th edn. Wiley, New York (1991)

19. Doǧru, O: On statistical approximation properties of Stancu type bivariate generalization of $q$-Balás-Szabados operators. In: Seminar on Numerical Analysis and Approximation Theory, pp. 179-194. Univ. Babeş-Bolya, Cluj-Napoca (2006)

20. Gadjiev, AD, Orhan, C: Some approximation theorems via statistical convergence. Rocky Mt. J. Math. 32(1), 129-138 (2002)

10.1186/1029-242X-2014-10

Cite this article as: Ren and Zeng: Some statistical approximation properties of Kantorovich-type $q$-Bernstein-Stancu operators. Journal of Inequalities and Applications 2014, 2014:10

Submit your manuscript to a SpringerOpen ${ }^{\circ}$ journal and benefit from:

- Convenient online submission

- Rigorous peer review

- Immediate publication on acceptance

Open access: articles freely available online

- High visibility within the field

- Retaining the copyright to your article

Submit your next manuscript at $\boldsymbol{s p r i n g e r o p e n . c o m ~}$ 